\title{
Research on Vehicle Detection Method based On LIDAR in UGV Leader-Follower Systems
}

\author{
Ziyong Jia ${ }^{a}$, Guoquan Ren ${ }^{b}$ and Dongwei $\mathrm{Li}^{\mathrm{c}}$ \\ Department of Vehicles and Electrical Engineer, Ordnance Engineering College, Shijiazhuang \\ 050003, China \\ a2432282264@qq.com, b876686117@qq.com, ‘632626442@qq.com
}

\begin{abstract}
Keywords: LIDAR, Clustering, Median filter, UGV Leader-follower Systems, Vehicle Detection.
\end{abstract}
\begin{abstract}
Aiming at the practical problem of target vehicle detection and tracking in the UGV Leader-follower Systems, a method of detecting and tracking vehicle by using LIDAR is proposed. As an active sensor, LIDAR can obtain the distance and angle information of the object in real time, and has high resolution and robustness. In this method, the LIDAR is installed on the following vehicle, and the two-dimensional laser scanning data in the environment is acquired in real time. Then the nearest neighbor clustering method is used to segment and cluster the LIDAR data. Then, the vehicle tail data is identified from the segmented data by using the straight line tail recognition method designed by the shape feature information of the vehicle, and the position information of the vehicle tail relative to the following vehicle is obtained. By using the data of LIDAR before and after the location of the contrast, determined the speed of the vehicle. Finally, the experiment proves that the method can meet the requirements of vehicle detection and tracking in the UGV Leader-follower Systems.
\end{abstract}

\section{Introduction}

With the development and accumulation of related technologies of the UGV, the related technology of the UGV leader-follower System has attracted much attention. The UGV leader-follower Systems refers to the multi-vehicle guided-following traffic pattern in the formation environment. It can be used for the formation of long-distance fleet under high-speed standard road. The UGV leader-follower System can reduce the burden of the driver effectively and reduce the danger on the long-distance driving process due to various types of complex factors. The main research contents of the UGV leader-follower System include the position and speed detection of pilot vehicle, and the control and path planning of vehicle following.

The pilot vehicle state detection is the basis and premise of the follower vehicle Self-follow in the UGV leader-follower System. At present, a variety of detection methods have been applied to the detection of vehicle, such as radar, vision cameras and ultrasound [1]. Haiyang Yuan[2], Fangfang $\mathrm{Hu}$ [3], Chenjiao Zhi [4] and so on presents a vehicle speed detection method based on machine vision, which is mainly used for vehicle speed detection under intelligent transportation system. Firstly, the vehicle is identified by the image processing method, and the vehicle speed is obtained by the video image processing technology. The algorithm is difficult to deal with the video image and the real-time property is difficult to be guaranteed. As an active sensor, the single-lane lidar can acquire the distance and angle information of the surrounding targets in real time, and has the advantages of all-weather, long-distance and good robustness. Daming Jiang [5] use the single-lane lidar for the UGCV navigation obstacle avoidance system, and to achieve local avoid obstacles. In this paper, we use the single-lane lidar to detect road features and establish a raster map from which we can determine the area through which the unmanned vehicles can pass. Xiuhua Zhuang [6] uses the single-line lidar to detect the obstacle in front of the vehicle through the area, and predict the position and direction of the dynamic obstacle. Zanfeng Luo [7], Can Wang [8] use multiple single-line laser radar scan different parts of the human body, through the generation of different characteristics to identify pedestrians.

In this experiment, the position and velocity of the pilot vehicle are detected by LIDAR. Firstly, the elevation angle of LIDAR is calibrated by trapezoidal calibration plate. The LIDAR data is segmented 
by the nearest neighbor method, and the isolated noise is effectively eliminated. The effect of the isolated noise is effectively eliminated and the effect of the isolated noise is effectively reduced. The median filtering of the clustering data can effectively remove the burst noise. The center of gravity and the range of the target pilot vehicle are extracted from the results of the data processing, and then the vehicle speed is calculated according to the comparison of the front and rear frame data.

\section{Installation and Calibration of LIDAR}

\subsection{The Selection of LIDAR and the Establishment of Coordinate.}

The LMS151 single-line LIDAR radar is used in this experiment. The measuring range is $0.1 \sim 50$ meters, the angle range is $270^{\circ}$, the angle resolution is $0.25^{\circ}$, the scanning frequency is $25 \mathrm{HZ}$.

The installation of the LIDAR on the vehicle body is a rigid connection. The position of the laser radar is fixed relative to the position and attitude of the vehicle body. Therefore, the position of the data points corresponding to the vehicle coordinate system is also fixed. The actual installation of the radar is shown in Fig 1. The position of the LIDAR is relative to the center of mass $(o, l, h)$ and the body coordinate system $\left(x_{\mathrm{veh}}, y_{\mathrm{veh}}, z_{\mathrm{veh}}\right),\left(x_{\mathrm{LiDAR}}, y_{\mathrm{LiDAR}}, z_{\mathrm{LiDAR}}\right)$, both in line with the right-hand rule.

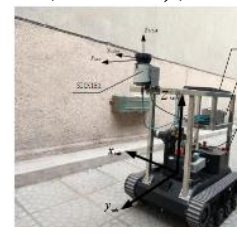

Fig. 1 The Lidar installated in the body

\subsection{Calibration of LIDAR External Parameters and Coordinate System Transformation.}

The LIDAR and the vehicle body are rigidly connected. The relative attitude and displacement of the LIDAR and vehicle are fixed. The arbitrary LIDAR scanning point has a unique point in the physical world. In the LIDAR scan image, only one pixel corresponding to object. Before the experiment, it is necessary to simply calibrate the installation of the lidar so that the data obtained by the LIDAR can be converted from the coordinate system of the LIDAR to the vehicle carrier coordinate system. During the experiment, in order to better achieve the purpose of vehicle detection, should minimize the impact of LIDAR roll angle. In the square area, the roll angle of the LIDAR is adjusted so that the LIDAR roll angle is approximately zero. Using the trapezoidal plate on the LIDAR calibration to determine the LIDAR pitch angle $\delta$, the principle shown in Fig 2.

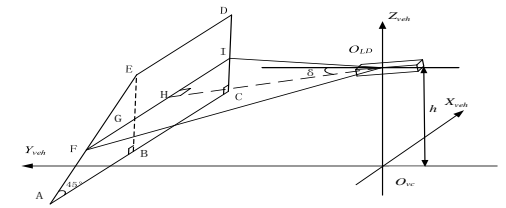

Fig. 2 Calibration Principle of LIDAR Pitch

Fie 2 shows the effect of the LIDAR scanning trapezoidal checkerboard calibration plate. $O_{\mathrm{LD}}$ is the optical center of the LIDAR, $O_{v e h}$ is the center of mass of the following vehicle, $\left(x_{v e h}, y_{v e h}, z_{v e h}\right)$ is the following vehicle coordinate system, $F I$ is the LIDAR radar trapezoid Board generated scan lines. $F G=E G, B G=B E-E G=B E-F G, F G=F I-B C$. Get the scan line in the relationship according to the figure:

$$
\begin{aligned}
& F I=\sqrt{\rho_{O_{L D}{ }^{2}+\rho_{O_{L D} I}{ }^{2}-2} \rho_{O_{L D} F} \rho_{O_{L D} I} \operatorname{COS}\left(\theta_{O_{L D} F}-\theta_{O_{L D} I}\right)} \\
& \delta=\arcsin \left(\frac{h-B G}{\rho_{O_{L D} I} \cdot \sin \left(\theta_{O_{L D} I}\right)}\right)
\end{aligned}
$$

The LIDAR data conversion to the reference coordinate system, the reference coordinate system is equivalent to the $\mathrm{x}$-axis rotation of the pitch angle $\delta$, coordinate transformation matrix:

$$
T_{x}=\left[\begin{array}{ccc}
1 & 0 & 0 \\
0 & \cos (\delta) & \sin (\delta) \\
0 & \sin (-\delta) & \cos (\delta)
\end{array}\right]
$$


Vertical type (1-2), establish the constraint equation, and then get the pitch angle $\delta$ of the LIDAR. According to the principle of laser radar imaging, the conversion model can be established:

$$
\left[\begin{array}{l}
x_{\text {veh }} \\
y_{\text {veh }} \\
z_{\text {veh }}
\end{array}\right]=\left[\begin{array}{ccc}
1 & 0 & 0 \\
0 & \cos (\delta) & \sin (\delta) \\
0 & \sin (-\delta) & \cos (\delta)
\end{array}\right]\left[\begin{array}{c}
\rho_{n} \cos \left(\theta_{n}\right) \\
\rho_{n} \sin \left(\theta_{n}\right) \\
0
\end{array}\right]+\left[\begin{array}{l}
0 \\
0 \\
h
\end{array}\right]=\left[\begin{array}{c}
\rho_{n} \cos \left(\theta_{n}\right) \\
\rho_{n} \sin \left(\theta_{n}\right) \cos (\delta) \\
-\rho_{n} \sin \left(\theta_{n}\right) \sin (\delta)+h
\end{array}\right]
$$

Where: $n$ is the LIDAR data sequence number, $\rho_{n}$ is the LIDAR scanning distance, $\theta_{n}$ is the initial scanning angle of the LIDAR, $h$ is the installation height of the laser radar, and $\delta$ is the laser radar pitch angle.

\section{Cluster Analysis and Median Filtering of LIDAR Data}

In order to obtain the contour features of the pilot vehicle from the LIDAR data, it is necessary to segment and cluster the data of the LIDAR data. In the process of LIDAR radar scanning, the return points of continuous obstructions are relatively dense, and there is a large gap between discontinuous obstructions. According to this feature, the neighbor points clustering analysis of LIDAR data is carried out. Aiming at the instability of the vehicle motion process, median filter algorithm is used to filter the laser radar data.

\subsection{Clustering Analysis of Adjacent Points.}

The purpose of clustering is to classify the detected objects, which is in accordance with the identification of the natural environment. The main idea of clustering is to use the first data point as the cluster center of the first group. If the distance from the next point to the point is less than the cluster radius $R$, then the data is put into this group Update to a new cluster center, otherwise the data as a new cluster center. The clustering algorithm in this paper adopts ABD algorithm:

$\left\|\rho_{i}-\rho_{i-1}\right\| \leq R$

$\rho_{i}$ is the distance of the current scan, $\rho_{i-1}$ is the distance of the previous scan detection point, and $\mathrm{R}$ is the radius of the segmentation.

If equation (1) holds, the two scanning points are the same obstacle. R:

$$
R=r_{i-1} \times \frac{\sin (\Delta \theta)}{\sin (\lambda-\Delta \theta)}+3 \delta
$$

From Fig.3, it is shown that the nearest neighbor clustering method has a good effect and provides clear environmental information for the following path planning and vehicle control. In the process of clustering, if the number of clustering points is only one. If it is greater than the threshold, the point is the noise point.
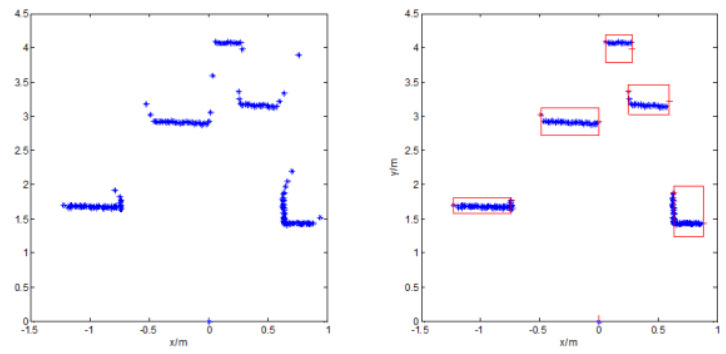

Fig. 3 Clustering analysis of obstacles

\subsection{Clustering point median filte.}

Ideally, a LIDAR is an isometric plane scan, and the scan data should be continuous. But in the process of moving vehicles, LIDAR will bump with the body. Different types of vehicles and road conditions, resulting in the performance of laser radar for different characteristics, especially in the off-road environment, the road is more complex, uneven road surface caused by vehicle bumps and the vehicle itself caused severe vibration of the LIDAR vibration, The scanning attitude angle will shift back and forth, making the scanning point of constant change, inevitably produce noise. 
The basic principle of median filtering is to replace the value of a point in a sequence with the median of each point in a neighborhood of the point, thus eliminating isolated noise points. The formula for median filter is:

$$
\rho_{i}=\operatorname{median}\left(\rho_{i-n}, \ldots, \rho_{i}, \ldots, \rho_{i+n}\right)
$$

Median filter effect depends on the size of the filter window, over the General Assembly makes the edge blurred, too small to denoise effect is poor. Select a different filter window, a group of experimental data were processed, the results shown in Fig 4.
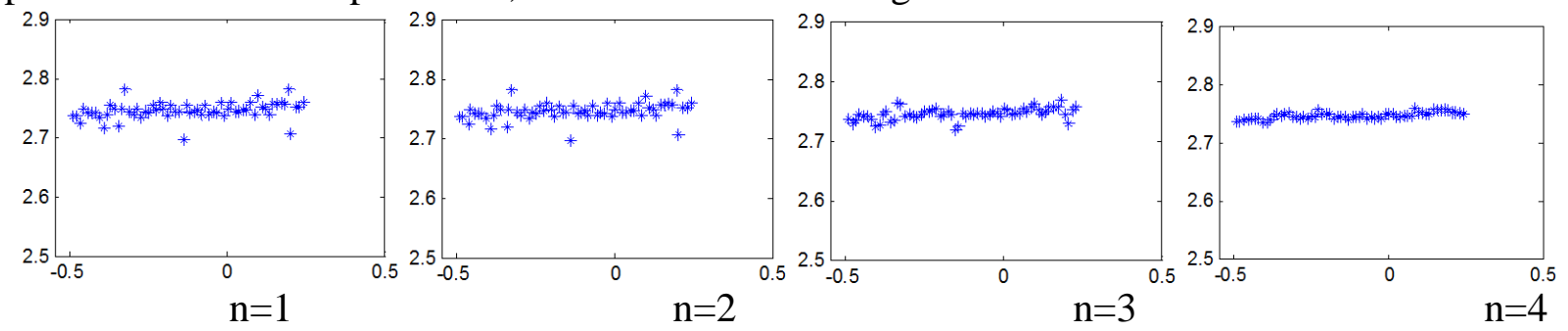

Fig. 4 Comparison of Median Filter Results of LIDAR Data Points

Through the comparative analysis of Figure 4 , the median filter window to take $n=3$ can achieve better filtering effect. Experiments show that median filtering can effectively remove the burst noise.

\section{Target Vehicle Detection and Tracking}

After the clustering process, the relative position and size of the targets in the range of LIDAR scanning are obtained. But in the tracking process need to prediction the movement of the car, it is necessary to get the lead relative speed and direction of information detection.

In the UGV Leader-follower Systems, the pilot vehicle detection parameters include position, speed, vehicle width and so on. $\left(C_{x}, C_{y}, v_{x}, v_{y}, \theta, l\right)$, where $\left(C_{x}, C_{y}\right)$ represents the state variables of the pilot vehicle in a two-dimensional raster map of the following vehicle, $\theta$ is the angle between the pilot vehicle heading and the following vehicle travel direction, and 1 is the width of the vehicle. In this experiment, the self-made crawler platform was selected in the pilot vehicle. The width of the vehicle was $0.8 \mathrm{~m}$, and the threshold range was $(0.7 \mathrm{~m}, 0.9 \mathrm{~m})$.

To further verify the feasibility of the speed detection algorithm, using Matlab to simulate the algorithm. First of all, outdoor experiments were carried out. First, the small-scale crawler platform equipped with LIDAR was at rest, and the data was obtained by the pilot's movement in front of the lidar. Figure 5 shows the left side of the six consecutive targets of the pilot vehicle scan points, through data processing, linear fitting effect as shown. Take the midpoint position as the center of gravity of the pilot, the position of the center of mass as shown in Figure 5. The position information of the first frame is the initial position, and the speed information of the pilot vehicle is continuously updated as shown on the right side of Fig 5. The detection of pilot vehicle position and speed provides information support for the follow-up vehicle's autonomous control.
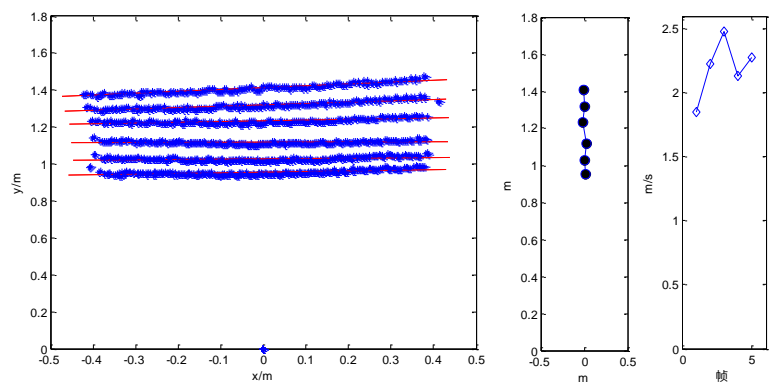

Fig. 5 Using MATLAB to process several consecutive frames of data 


\section{Summary}

In this paper, we mainly study the speed detection algorithm of target pilot vehicle based on the single-lane LIDAR, which is based on the autonomous follower application of unmanned vehicles. In order to obtain the distance and position information of the vehicle using LIDAR, the LIDAR is first calibrated and the LIDAR data is converted to the following body coordinate system for analysis. Then the nearest neighbor method is used to cluster the LIDAR data, and median filtering is applied to the clustered data, which effectively removes the burst noise. Through data processing, determine the location of the vehicle. By comparing the data of the front and rear frames of the LIDAR, the vehicle speed is determined, which provides the information support for the follow - up vehicle 's autonomous control. The experimental results show the feasibility and effectiveness of the proposed algorithm. However, this experiment only considers the detection of vehicle position and speed, and does not consider the recognition of vehicles. The future work will be based on visual and single-lane lidar fusion of information for the direction of development, in order to achieve accurate identification of vehicles, and to detect the location and speed of the vehicle.

\section{References}

[1]. LI W, ZHANG R, LIU Z K, et al. An approach of laser-based vehicle monitor [J], Applied Mathematics and Computer, 2007, 185(2):953-962.

[2]. Min,Choi J.Vehicle Positioning Technology Using Infra-based Laser Scanner Sensors for Autonomous Driving Service Computer Science and Convergence[M].Springer Netherlands, 2012, 114:511-517.

[3]. Khatib O.Real -Time Obstacle Avoidance for Manipulators and Mobile Robpts[C].The Int. J.Robot. Res, 2010, Vol 5, pp. 90-98.

[4]. X.Yuan, C.X.Zhao,H.F. Zhang. Road Detection and Corner Extraction Using High Definition Lidar. Information Technology Journal, 2010, 9(2):1022-1030.

[5]. Kinjal A Joshi, Darshak G Thakore. A Survey on Moving Object Detection and Tracking in video Surveillance System [J].International Journal of Computing and Engineering (IJSCE): 2012 (2): 44-48.

[6]. LUO Zanfeng. Pedestrain Trackng Based on Multiple Laser Scanners [D].Hangzhou: Zhejiang University, 2012(8):25-28.

[7]. Wang J X, Jin X F.Moving Object Detection and Tracking in Mobile Robot System Based on Omni-Visionl and Laser Rangefinder[C]//Recent Advences in CSIE 2011,2012:133-139.

[8]. LIU Daxue, DAI Bin, LI Zheng, et al. A method for calibration of single line laser radar and camera [J]. Journal of Huazhong University of Science and Technology: Natural Science Edition, 2008, 36 (Supplementary I):68-71. 